\title{
Resource Allocation in downlink of LTE using Bandwidth prediction through Statistical Information
}

\author{
S.Gayathri ${ }^{1}$, R.Sabitha ${ }^{2}$ \\ ${ }^{1}$ Research Scholar, Sathyabama University, OMR Road, Chennai-119, India \\ ${ }^{2}$ Principal, and Head, Department of Information Technology, Jeppiaar Engineering College, OMR Road, \\ Chennai-119, India
}

\begin{tabular}{|c|c|}
\hline Article Info & ABSTRACT \\
\hline Article history: & Long Term Evolution (LTE) is the technology used in modern third and \\
\hline Received Nov 19, 2017 & $\begin{array}{l}\text { fourth generation mobile wireless cellular networks. Due to the presence of } \\
\text { large number of users, mobility and varying channel conditions, proper }\end{array}$ \\
\hline Revised Jan 23, 2018 & resource allocation is essential to provide a good user experience and \\
\hline Accepted Feb 19, 2018 & $\begin{array}{l}\text { improve the system throughput. In this paper, a resource allocation algorithm } \\
\text { is implemented that will use the probabilistic models to predict the channel }\end{array}$ \\
\hline Keywords: & $\begin{array}{l}\text { condition and allocate resources accordingly. Also, the algorithm will } \\
\text { support QoS requirements. During the resource allocation, the channel }\end{array}$ \\
\hline LTE & quality information is collected and analyzed to predict the future channel \\
\hline OFDMA & conditions and resource allocation vectors are configured accordingly. The \\
\hline Resource Allocation & performance of the algorithm is analyzed based upon the data collected. The \\
\hline Bandwidth & algorithm is able to provide a reasonable success rate for channel prediction. \\
\hline Throughput & $\begin{array}{l}\text { By using the resource allocation vectors and channel prediction, the } \\
\text { algorithm performance also is improved considerably due to the lesser space } \\
\text { and time complexity required }\end{array}$ \\
\hline
\end{tabular}

Copyright $\odot 2018$ Institute of Advanced Engineering and Science. All rights reserved.

\section{Corresponding Author:}

S.Gayathri,

Department of Computer Science and Engineering,

Sathyabhama University,

OMR Road, Chennai-119, India.

Email: gayathri.nishitha@gmail.com

\section{INTRODUCTION}

Modern mobile and wireless cellular networks use the LTE as the technology for communication. It is used in third and fourth generation mobile networks and replaces the GSM/CDMA technologies as the core technology for these generations.

LTE uses Orthogonal Frequency Division Multiplexing (OFDMA) as the access scheme in downlink side and Single Carrier Frequency Division Multiple Access (SC-FDMA) as the access scheme for uplink side. In OFDMA, a high frequency carrier is divided into several low frequency subcarriers with smaller bit rates. The division of carriers is such that the sub-carrier are orthogonal to each other to eliminate inter carrier interference. Also a smaller guard interval will be placed between the sub carriers to prevent inter symbol interference. SC-FDMA is a customized version of OFDMA with throughput comparable to that of OFDMA. Inter Symbol Interference may be caused in SC-FDMA due to multipath propagation which is handled by base station using adaptive frequency domain equalization.

Due to the widespread use of the mobile cellular networks, high bandwidth and throughput are required in today's LTE networks. To achieve this, proper resource allocation has to be implemented. The resource allocation in LTE is performed at Medium Access Control (MAC) layer. Due to the nature of the LTE networks, large number of users, mobility of users, varying channel conditions, high bandwidth requirements, inter channel interference can affect the network performance and throughput and hence proper resource allocation is required to optimize the network performance. Besides, sudden changes in 
channel conditions could affect resource allocation approaches considerably since it will be difficult to have a stable prediction of channel conditions. Requirements like Voice over LTE (VoLTE) and audio/video streaming impose their own real-time performance requirements which have to be handled to provide proper user experience.

A radio resource scheduling algorithm for video streaming is discussed in [1]. It proposes a packetlevel service differentiation to improve the throughput. Resource allocation techniques using carrier aggregation is discussed in [2], [3] and [4]. It uses the channel quality information and user profile to improve the resource allocation. Cross layer algorithms which optimize the resource allocation over LTE systems are discussed in [5] and [6]. They use the information from application, MAC and physical layers to optimize the resource allocation algorithms.

A frame based weighted fair queuing algorithm which tries to improve the spectral efficiency in OFDMA networks is discussed in [7]. A resource allocation algorithm that tries to both optimize the throughput and achieve fairness is discussed in [8]. It tries to service the nodes with good channel condition as well as the nodes with poor channel conditions by prioritizing both the channel conditions and fairness. An algorithm to provide utility proportional fairness which prevents fluctuations in resource allocation under both high and low traffic conditions is discussed in [9]. The performance of the frequency domain packet scheduling and the impact of the inter cell interference is discussed in [10].

Radio resource allocation in autonomous devices using LTE for machine to machine communications is discussed in [11]. Here attempts are made to share the radio resources efficiently to reduce inter-channel interference and improve network efficiency. A resource allocation algorithm for users with different QoS constraints based upon queuing systems is discussed in [12]. It proposes a G/M/1 queuing system to model the users with different QoS constraints into different queues. An inter cell interference avoidance scheme using active coordination between cells is discussed in [13]. A multiuser scheduling algorithm that tries to maximize throughput while maintaining fairness is discussed in [14]. It uses sum utility maximization along with fairness levels to satisfy the nodes with poor channel conditions.

A QoS aware packet scheduling algorithm that both tries to satisfy QoS requirements as well as providing fairness to nodes with poor channel conditions is discussed in [15]. A multi-level queue based resource allocation algorithm in downlink of OFDMA networks is discussed in [16]. Various OFDMA scheduling algorithms are discussed in [16], [17] and [18]. LTE scheduling algorithms are discussed in [19], [20] and [21]. In [22] discussed the privacy-preserving methods was vulnerable to Sybil attacks, whereby a malicious user can act as to be multiple (other) vehicles. It ensured the authenticity of the messages propagated in VANET; a straight-forward process is utilized public keys authorized by a certification authority (CA) to sign the messages. Fadli Sirait [23] developed an Ethernet over SDH (synchronous digital hierarchy) and MPLS-TP (Multiprotocol Label Switching - Transport Profile) with ring security to predictable network failure and computed performance of network. Aman et al [24] integrated context transport and multicast quick reroute, and developed this integration to the standard network mobility management. Hashim et al. [25] discussed quantitative analysis and it computed Proxy Mobile Internet Protocol Version 6 (PMIPv6) multicast speedy reroute operations for data traffic.

In this paper, a resource allocation algorithm is implemented using the statistical information for the channel conditions to predict the channel conditions and perform resource allocation based upon the channel conditions.

Section 2 lists the various resource allocation approaches already done in the field. Section 3 describes the resource allocation algorithm in detail. Section 4 provides details about the results collected and the algorithm performance. Section 5 provides the future improvements which can be made in the algorithm.

\section{RESEARCH METHOD}

A LTE network consists of Evolved UMTS Terrestrial Random Access Network (E-UTRAN), Evolved Packet Core (EPC), several User Equipment (UE) and Packet Data Network (PDN) Gateway. An EUTRAN consists of one or more base stations called evolved NodeB or eNodeB and user equipments (UE). The eNodeB communicates to UEs using OFDMA for downlink and SC-FDMA for uplink.

EPC consists of Mobility Management Entity (MME), Serving Gateway (S-GW), Packet Data Network Gateway (PDN-GW), Home Subscriber Server (HSS), Policy Control and Charging Rules Function (PCRF). PGN Gateway communicates with the outside world including IPv4/IPv6 networks. The Serving gateway acts as a router forwarding data between base stations and the PDN gateway. Home Subscriber Server has the database containing information about the all the subscribers connected to the network.

Mobility Management Entity controls the operation of the User Equipments through HSS and various control signals. The Policy Control and Charging Rules Function (PCRF) is responsible for policy control decision making, controlling the flow-based charging in Policy Control Enforcement Function 
(PCEF). Let us assume a LTE network with a base station and N number of nodes. The number of nodes will vary depending dynamically as the users come in and go out of the network randomly. At any point of time, different number of nodes will have different channel conditions, bandwidth requirements and QoS requirements. The channel condition of a node can vary over time due to the mobility of nodes, interference between the nodes and inter cell interference.

The nodes can be split into separate set of groups based upon the QoS requirements and channel conditions. All the nodes with QoS requirements can be kept in one or more groups depending on the variations in channel conditions and QoS requirements. The nodes without QoS requirements can be kept in one or more groups depending on the channel conditions. Such partitioning can break the larger resource allocation problem into a set of smaller allocation problem which helps improving the performance of the resource allocation algorithm.

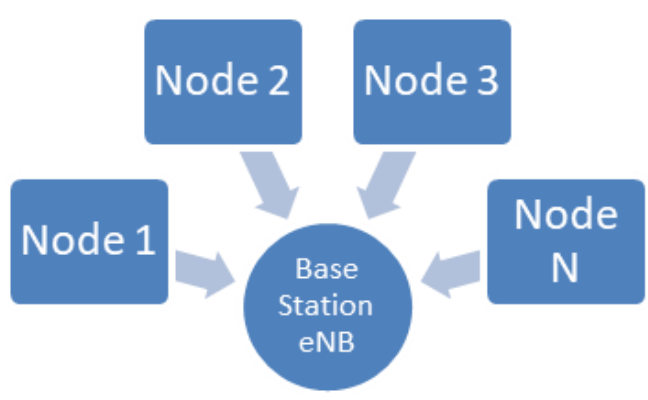

Figure 1. Block Diagram of LTE cell

Since calculating the statistical parameters for each allocation of resources can be tedious and time consuming, a resource allocation vector can be created periodically which can be used to generate the resource allocation for next I iterations. Doing so reduces the requirements for calculations for each iteration of algorithm as well as reordering of queues each time reducing the performance requirements considerably. While there is a drawback in this approach that it will prevent instantaneous responses to the changes in channel conditions, such prevention could improve the performance if such changes are only momentary and the nodes may get back again to same condition.

The algorithm calculates the statistical mean, variance and standard deviation for the nodes periodically and uses them to predict the future channel conditions and generates the allocation vectors accordingly. If the period for each iteration of algorithm is $\mathrm{T}$, nodes may have more transmission opportunities depending upon their QoS requirements and channel conditions. Nodes with good channel conditions and QoS requirements will have more transmission opportunities comparing to the nodes without QoS requirements and poor channel conditions. If nodes have poor channel conditions but have QoS requirements, effort is made to make sure they have QoS requirements, effort is made to make sure they receive enough transmission opportunities to achieve as much throughput as possible without affecting the throughput of other nodes. For nodes with poor channel conditions, effort is made to ensure that they receive at least a minimal quantity of transmission opportunities to ensure fairness.

Following is the algorithm to perform the resource allocation:

Step 1. Initialization of network:

NodeList $\mathrm{N}=$ empty

Overall network throughput $\mathrm{T}_{\mathrm{N}}=0$

Overall transmitted bytes $\mathrm{T}_{\mathrm{B}}=0$

Step 2. Initialization of node(s):

QoS requirements $\mathrm{N}_{\mathrm{QOS}}=$ ExpectedQoSThroughput

ActualThroughput $\mathrm{N}_{\mathrm{T}}=0$

StartTime $\mathrm{N}_{\mathrm{ST}}=0$

TotalTransmittedBytes $\mathrm{N}_{\mathrm{B}}=0$

Mean $\mathrm{N}_{\mathrm{M}}=0$

Variance $\mathrm{N}_{\mathrm{V}}=0$

Standard Deviation $\mathrm{N}_{\mathrm{SD}}=0$

List of nodes $\mathrm{N}+=$ Current Node $\mathrm{N}$

If node is QoS aware, 
Move into QoS aware node list. Partition the list based upon the channel conditions and QoS requirements

If node is non-QoS aware,

Move into non-QoS aware node list and partition the list based upon the channel condition and QoS requirements

Step 3. Calculation of various parameters:

During each iteration of resource allocation, for each node which has transmission opportunity:

$\mathrm{N}_{\mathrm{B}}+=$ Transmitted Bytes

During each iteration of algorithm, for each node,

Time Elapsed $\mathrm{T}_{\mathrm{E}}=\mathrm{N}_{\mathrm{L}}-\mathrm{N}_{\mathrm{S}}$

Mean $\mathrm{T}_{\mathrm{M}}=\mathrm{T}_{\mathrm{B}} / \mathrm{T}_{\mathrm{E}}$

For each node,

Variance $\mathrm{N}_{\mathrm{V}}=\mathrm{N}_{\mathrm{T}}-\mathrm{T}_{\mathrm{M}}$

Standard Deviation $\mathrm{N}_{\mathrm{SD}}=\sqrt{\mathrm{N}_{\mathrm{V}}}{ }^{2} \mathbb{N}$

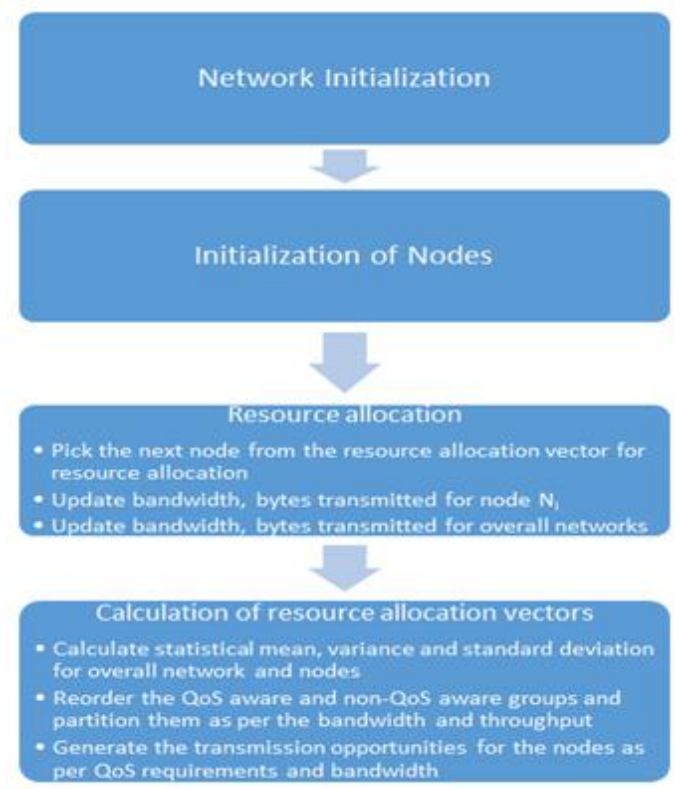

Figure 2. Resource Allocation Algorithm

Step 4. Generation of resource allocation vectors:

$\mathrm{N}_{\mathrm{R}}=$ number of resource allocations

$\mathrm{N}_{\min }=$ minimum number of resource allocations

For each node, predicted bandwidth is

$$
\mathrm{N}_{\mathrm{B}}=\mathrm{N}_{\mathrm{M}} \pm \mathrm{N}_{\mathrm{SD}}
$$

In the QoS aware node list, order the nodes based upon the calculated $\mathrm{N}_{\mathrm{B}}$ and partition the list

In the Non-QoS aware node list, order the nodes based upon the calculated $\mathrm{N}_{\mathrm{B}}$ and partition the list.

Let $\mathrm{N}_{\mathrm{ra}}$ is the transmission opportunities available for generation of resource allocation vector.

Let $\mathrm{T}_{\min }$ be the minimum bandwidth that is guaranteed for the nodes without QoS requirements.

Then the transmission opportunities for the non-QoS nodes will be at least $\mathrm{T}_{\min } / \mathrm{B}_{\text {pre. }}$

Now remaining transmission opportunities is

$$
\mathrm{N}_{\mathrm{ra}}-\sum \mathrm{T}_{\min } / \mathrm{B}_{\text {pre. }}
$$

For QoS aware nodes, calculate the maximum transmission opportunities required for each node to satisfy the QoS requirements.

Calculate the difference between required quantity of transmission opportunities and the available quantity

Difference $\mathrm{D}=\mathrm{T}_{\mathrm{xo}}$ Required $-\mathrm{T}_{\mathrm{xo}}$ Available

If $\mathrm{D}$ is negative, we have excess transmission opportunities available. This can be shared between the nodes proportionally. 
If $\mathrm{D}$ is positive, we have lesser transmission opportunities available. This can be shared between QoS aware nodes proportionally.

\section{RESULTS AND ANALYSIS}

The algorithm has been implemented using NS3 simulator and the results have been collected. The performance has been analyzed. There is considerable improvement in case of CPU utilization since the resource allocation vectors are not calculated every time. The following table shows some of the resource allocation occurrences for different nodes.

Table 1. Resource Allocation Values

\begin{tabular}{ccc}
\hline Time & Node & Bytes allocated \\
\hline 0.385 & 15 & 2196 \\
0.386 & 25 & 2196 \\
0.387 & 36 & 2196 \\
0.388 & 37 & 2196 \\
0.389 & 41 & 2196 \\
0.390 & 42 & 2196 \\
0.391 & 43 & 2196 \\
0.392 & 2 & 2196 \\
0.393 & 1 & 2196 \\
0.394 & 11 & 2196 \\
\hline
\end{tabular}

The results are shown in the following graph:

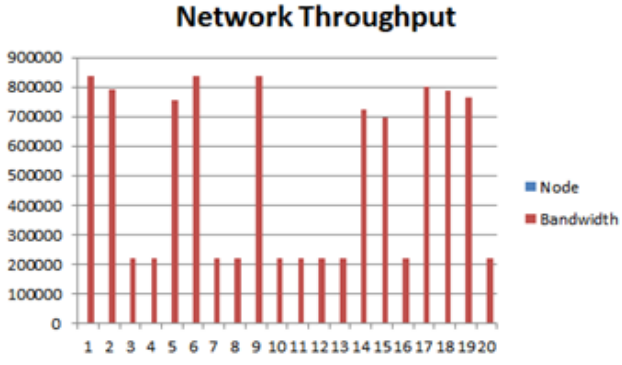

Figure 3. Network throughput using the Algorithm

Following chart shows the performance of the algorithm over time. Here, the nodes with QoS requirements and good channel conditions obtain better throughput since the allocation is favorable for them. Nodes with QoS requirements but poor channel conditions get relatively lesser throughput since despite they are allocated favorably, the poor channel conditions prevent them from achieving the optimal bandwidth. In case of non-QoS aware nodes, they get almost constant minimal bandwidth provided the channel quality doesn't change over time. Otherwise, they get bandwidth as per the underlying channel conditions. Also, please note that as number of nodes increases in the network, the allocation to a specific node decreases accordingly to maintain the balance in resource allocation between various nodes.

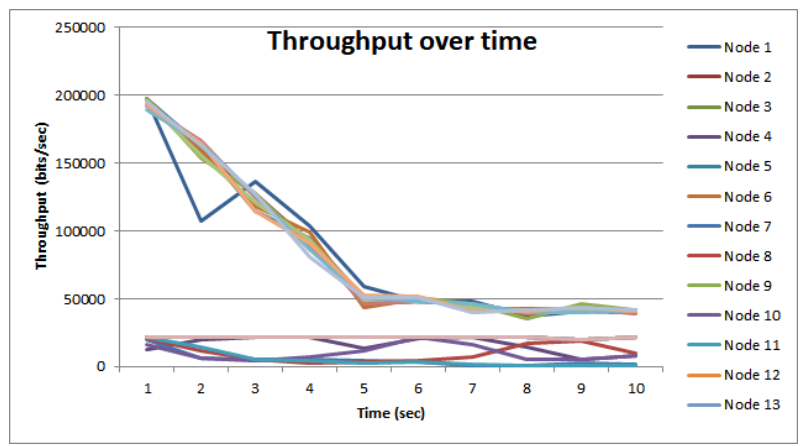


Figure 4. Performance over time

Following chart shows the comparison of algorithm performance to that of Round Robin or Proportional Fair scheduling algorithms.

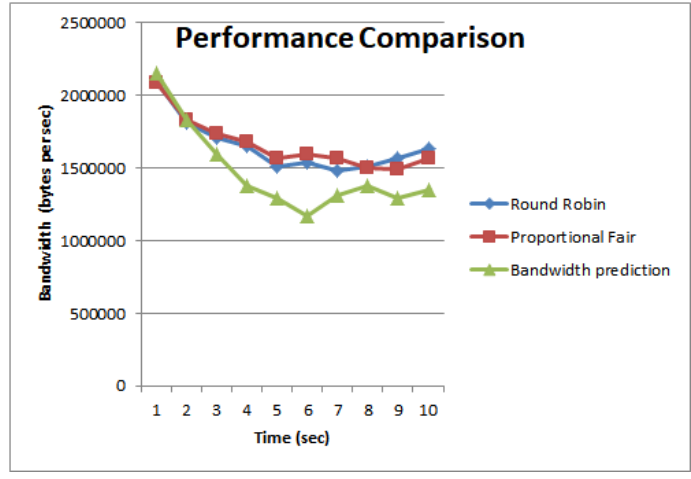

Figure 5. Performance comparison

As seen in the chart, the algorithm throughput will be relatively lesser than that of the other two algorithms. The reason is that some of the nodes for which channel condition is poor, due to QoS requirements, more allocation opportunities have to be provided which will cause the algorithm throughput to fall below that of other two algorithms. This is acceptable since attempt is made to satisfy the QoS requirements in a best effort basis. If the channel condition is good or nodes specifying QoS requirements have good channel condition, the algorithm performance is as good as that of other algorithms.

It has to be noted that while the prediction of the network channel conditions is good for nodes which doesn't have momentary channel fluctuations, for the nodes having momentary channel fluctuations, the prediction suffers briefly sometimes. But once the node stabilizes, the algorithm behavior returns back to normal.

Since the calculation of allocation vectors is done not every time but once in $\mathrm{N}$ iterations only, the performance of the algorithm is in the range of $\mathrm{O}(1)$ in general and $\mathrm{O}(\mathrm{n} \log \mathrm{n})$ for the particular iteration. Since the generation of the allocation vector and ordering of the nodes based upon the channel conditions is done in place, no separate iteration of the list of nodes will be required thereby improving the performance considerably.

\section{CONCLUSION}

The resource allocation algorithm which predicts the bandwidth based upon the statistical data has been implemented and its performance has been verified using the simulation results. The algorithm improves the network throughput and CPU utilization considerably. In case of sudden changes in signal conditions, this sometimes affects the prediction of bandwidth and hence algorithm performance momentarily.

\section{REFERENCES}

[1] Wen-Ping Lai, En-Cheng Liou, and Ping-Chi Chen., "Radio resource scheduling using packet-level service differentiation for video over the LTE downlink", IEEE/CIC International Conference on Communications in China (ICCC), pp.851-855, Oct 2014.

[2] Husnu S. Narman, Mohammed Atiquzzaman, "Joint and partial carrier components assignment techniques based on user profile in LTE systems”, Wireless Communications and Networking Conference (WCNC), Mar 2015, pp. 983-988.

[3] Pei-Ling Tsai, Kate Ching-Ju Lin and Wen-Tsuen Chen, "Downlink radio resource allocation with Carrier Aggregation in MIMO LTE-advanced systems", IEEE International Conference on Communications (ICC), Jun 2014, pp. 2332-2337.

[4] Ahmed Abdelhadi and Charles Clancy, "An optimal resource allocation with joint carrier aggregation in 4GLTE”, International Conference on Computing, Networking and Communications (ICNC), Feb 2015, pp. 138142. 
[5] Sotiris Karachontzitis, Tasos Dagiuklas and Lambros Dounis, "Fair cross-layer scheme for heterogeneous H.264/AVC video streams over LTE-based broadband systems", 8th International Wireless Communications and Mobile Computing Conference (IWCMC), Aug 2012, pp. 1006-1010.

[6] Dabing Ling, Zhaoming Lu, Wei Zheng, Xiangming Wen and Ying Ju, "Energy efficient cross-layer resource allocation scheme based on potential games in LTE-A", 15th International Symposium on Wireless Personal Multimedia Communications (WPMC), pp. 623-627, Sep 2012.

[7] Nguyen Huu Thanh, Nguyen Van Duc, Le Thi Hang, Do Hanh, Nguyen Tai Hung and Tran Anh Tu, "On providing fairness, QoS support and spectral efficiency in OFDMA/TDD environments", International Conference on Information Networking (ICOIN), Jan 2011, pp. 218-223.

[8] Shih-Jung Wu and Liou Chu, "A Novel Packet Scheduling Scheme for Downlink LTE System”, Seventh International Conference on Intelligent Information Hiding and Multimedia Signal Processing (IIH-MSP), Oct 2011, pp. 25-28.

[9] Ahmed Abdel-Hadi and Charles Clancy, "A robust optimal rate allocation algorithm and pricing policy for hybrid traffic in 4G-LTE”, 24th International Symposium on Personal Indoor and Mobile Radio Communications (PIMRC), Sep 2013, pp. 2185-2190.

[10] A. Pokhariyal, G. Monghal, K. I. Pedersen, P. E. Mogensen, I. Z. Kovacs, C. Rosa and T. E. Kolding, "Frequency Domain Packet Scheduling Under Fractional Load for the UTRAN LTE Downlink", Vehicular Technology Conference, 2007. VTC2007-Spring. IEEE 65th, Apr 2007, pp. 699-703.

[11] Adnan Aijaz and A. Hamid Aghvami, "On Radio Resource Allocation in LTE Networks with Machine-toMachine Communications”, Vehicular Technology Conference (VTC Spring), 2013 IEEE 77th, Jun 2013, pp. $1-5$.

[12] Mohsin Iftikhar, Nada Al Zaben and Suparerk Manitpornsut, "The most promising scheduling algorithm to provide guaranteed QoS to all types of traffic in multiservice 4G wireless networks", International Joint Conference on Computer Science and Software Engineering (JCSSE), Jun 2012, pp. 109-115.

[13] Ján Kulík and Peter Farkaš, "Inter-cell interference avoidance by active coordination of scheduling for downlink on LTE like systems", 22nd International Conference on Applied Electromagnetics and Communications (ICECOM), Sep 2016, pp. 1-5.

[14] Stefan Schwarz, Christian Mehlfuhrer and Markus Rupp, "Throughput Maximizing Multiuser Scheduling with Adjustable Fairness", IEEE International Conference on Communications, Jun 2011, pp. 1-5.

[15] Alexandru Vulpe, Simona Halunga and Octavian Fratu, "QoS-aware downlink scheduling in multi-carrier communication systems", 4th International Conference on Wireless Communications, Vehicular Technology, Information Theory and Aerospace \& Electronics Systems, May 2014, pp. 1-5.

[16] S.Gayathri and R.Sabitha, "Multi-Level Queue based Resource Allocation in Downlink of OFDMA Wireless Cellular Networks", Indian Journal of Science and Technology, vol. 9, no. 10, March 2016.

[17] Wong, I.C., Cellular Products Group R\&D, Freescale Semicond., Inc., Austin, TX ; Evans, B.L., "Adaptive downlink OFDMA resource allocation”, Signals, Systems and Computers, 2008 42nd Asilomar Conference, Oct 2008, pp.2203-2207.

[18] Guowang Miao; Georgia Inst. of Technol., Atlanta; Himayat, N., "Low Complexity Utility Based Resource Allocation for 802.16 OFDMA Systems", Wireless Communications and Networking Conference, 2008. WCNC 2008, Apr 2008, pp.1465-1470.

[19] Barakat, B.; Dept. of Electr., Electron. \& Comput. Eng., Univ. of Greenwich, London, UK; Arshad, K., “An adaptive hybrid scheduling algorithm for LTE-Advanced", Telecommunications (ICT), 2015 22nd International Conference, April 2015, pp.91-95.

[20] F.Capozzi, G.Piro, L.A. Grieco, G.Boggia, P.Camarda, "Downlink Packet Scheduling in LTE Cellular Networks: Key Design Issues and a Survey", IEEE Comm. Surveys and Tutorials, vol. 15, no. 99, pp.1-23, Jun. 2012.

[21] Stefan Schwarz, Christian Mehlf uhrer and Markus Rupp Institute of Communications and Radio-Frequency Engineering, Vienna University of Technology Gusshausstrasse 25/389, A-1040 Vienna, Austria, Mehlfuhrer, C.; Rupp, M., "Low Complexity Approximate Maximum Throughput Scheduling for LTE”, 2010 Conference Record of the Forty Fourth Asilomar Conference on Signals, Systems and Computers (ASILOMAR), , Nov 2010, pp.1563-1569.

[22] T. Hemalatha, "SECURE Detecting Sybil Attack with a Scalable Protocol", International Journal of MC Square Scientific Research, vol. 4, no. 1, pp. 35-41, 2012.

[23] Fadli Sirait., "Capacity Improvement and Protection of LTE Network on Ethernet Based Technique", TELKOMNIKA (Telecommunication Computing Electronics and Control), vol. 16, no.1, pp. 1-10, 2018.

[24] Aman, A. H. M., Hashim, A. H. A., \& Ramli, H. A. M., "Simulation Analysis for Multicast Context Delivery Network Mobility Management", Indonesian Journal of Electrical Engineering and Informatics (IJEEI), vol. 5, no. 4, pp. 390-394, 2017.

[25] Hashim, A. H. A., Aman, A. H. M., \& Ramli, H. A. M., "Quantitative Evaluation for PMPIv6 Multicast Fast Reroute Operations", Bulletin of Electrical Engineering and Informatics (BEEI), vol. 6, no. 4, pp. 371-376, 2017. 